\title{
A mean redshift of 2.8 for Swift gamma-ray bursts
}

\author{
P. Jakobsson ${ }^{1}$, A. Levan ${ }^{2,3}$, J. P. U. Fynbo ${ }^{1}$, R. Priddey ${ }^{2}$, J. Hjorth ${ }^{1}$, N. Tanvir ${ }^{2}$, D. Watson ${ }^{1}$, B. L. Jensen ${ }^{1}$, \\ J. Sollerman ${ }^{1}$, P. Natarajan ${ }^{4}$, J. Gorosabel ${ }^{5}$, J. M. Castro Cerón ${ }^{1}$, K. Pedersen ${ }^{1}$, T. Pursimo ${ }^{6}$, A. S. Árnadóttir ${ }^{3}$, \\ A. J. Castro-Tirado ${ }^{5}$, C. J. Davis ${ }^{7}$, H. J. Deeg ${ }^{8}$, D. A. Fiuza ${ }^{9}$, S. Mykolaitis ${ }^{10}$, and S. G. Sousa ${ }^{11,12}$
}

${ }^{1}$ Dark Cosmology Centre, Niels Bohr Institute, University of Copenhagen, Juliane Maries Vej 30, 2100 Copenhagen, Denmark e-mail: pallja@astro.ku.dk

${ }^{2}$ Centre for Astrophysics Research, University of Hertfordshire, College Lane, Hatfield, Herts AL10 9AB, UK

${ }^{3}$ Lund Observatory, Box 43, 22100 Lund, Sweden

${ }^{4}$ Department of Astronomy, Yale University, PO Box 208101, New Haven CT 06520-8101, USA

5 Instituto de Astrofísica de Andalucía (CSIC), Apartado de Correos, 3004, 18080 Granada, Spain

${ }^{6}$ Nordic Optical Telescope, Apartado de Correos, 474, 38700 Santa Cruz de la Palma (Tenerife), Spain

7 Joint Astronomy Centre, University Park, 660 North A'ohoku Place, Hilo, HI 96720, USA

8 Instituto de Astrofísica de Canarias, c/. Vía Láctea, s/n, 38200 La Laguna (Tenerife), Spain

9 Observatory, University of Helsinki, PO Box 14, 00014 University of Helsinki, Finland

10 Institute of Theoretical Physics and Astronomy, A. Gostauto St. 12, 01108 Vilnius, Lithuania

11 Centro de Astrofísica da Universidade do Porto, Rua das Estrelas, 4150-762 Porto, Portugal

12 Centro de Astrofísica Observatório Astronómico de Lisboa, Tapada da Ajuda, 1349-018 Lisboa, Portugal

Received 30 September 2005 / Accepted 21 October 2005

\section{ABSTRACT}

The exceptionally high luminosities of gamma-ray bursts (GRBs), gradually emerging as extremely useful probes of star formation, make them promising tools for exploration of the high-redshift Universe. Here we present a carefully selected sample of Swift GRBs, intended to estimate in an unbiased way the GRB mean redshift $\left(z_{\text {mean }}\right)$, constraints on the fraction of high-redshift bursts and an upper limit on the fraction of heavily obscured afterglows. We find that $z_{\text {mean }}=2.8$ and that at least $7 \%$ of GRBs originate at $z>5$. In addition, consistent with pre-Swift observations, at most $20 \%$ of afterglows can be heavily obscured. The redshift distribution of the sample is qualitatively consistent with models where the GRB rate is proportional to the star formation rate in the Universe. We also report optical, near-infrared and X-ray observations of the afterglow of GRB 050814, which was seen to exhibit very red optical colours. By modelling its spectral energy distribution we find that $z=5.3 \pm 0.3$. The high mean redshift of GRBs and their wide redshift range clearly demonstrates their suitability as efficient probes of galaxies and the intergalactic medium over a significant fraction of the history of the Universe.

Key words. cosmology: observations - dust, extinction - early Universe - galaxies: high-redshift - gamma rays: bursts

\section{Introduction}

The potential of long-duration ( $>2 \mathrm{~s}$ ) gamma-ray bursts (GRBs) as probes of the high-redshift Universe has long been recognised. The immense luminosities of the bursts, coupled with their origin in the core collapse of massive stars (Hjorth et al. 2003a; Stanek et al. 2003) and $\gamma$-ray penetration through dust, suggests a variety of intriguing applications. Much effort has been directed into the use of bursts for studying star formation (e.g. Fruchter et al. 1999; Christensen et al. 2004; Tanvir et al. 2004; Jakobsson et al. 2005a), as backlights for exploring high-redshift galaxies and the intergalactic medium (e.g. Vreeswijk et al. 2004; Jakobsson et al. 2004a) and even as probes of cosmological parameters (e.g. Ghirlanda et al. 2004;
Mörtsell \& Sollerman 2005). Although the GRB population observed until the end of 2004 had enabled much progress in the field, it was widely expected that the launch of Swift, and the subsequent order of magnitude increase in the number of GRBs open to detailed study, would allow further insight into the high-redshift Universe (Gehrels et al. 2004). Indeed, the ability of Swift to locate and follow-up a fainter burst population than was previously possible (Berger et al. 2005b) has allowed more distant bursts to be studied. The mean redshift of pre-Swift bursts was $z_{\text {mean }}=1.4$, while bursts discovered by Swift now have $z_{\text {mean }}=2.8$, including the first burst to have been discovered with $z>6$, GRB 050904 at $z=6.29$ (Haislip et al. 2005; Kawai et al. 2005; Price et al. 2005; Tagliaferri et al. 2005: Watson et al. 2005b). 
High-redshift bursts, whose afterglows may already be faint due to the large luminosity distance ${ }^{1}$ (e.g. GRB 020124: Berger et al. 2002; Hjorth et al. 2003b) are rendered essentially invisible at optical wavelengths for $z>6$ due to hydrogen opacity when the redshifted Ly $\alpha$ break sweeps through the optical regime; an effect that is clearly seen in galaxies in the Hubble Deep Field (e.g. Spinrad et al. 1998; Weymann et al. 1998). Therefore, a simple diagnostic of a high-redshift burst is its absence in deep optical observations and detection in a redder filter. Such a detection does not unambiguously fix the redshift, since high local extinction $\left(A_{V}\right)$ will also markedly reduce the short wavelength flux. However, with multi-wavelength observations it is possible to distinguish the curved red spectrum expected for an extinguished burst, from the sharp cut-off due to the Ly $\alpha$ break. This method is similar to that which has been used successfully (and accurately) in the selection and study of Lyman-break galaxies (Steidel \& Hamilton 1992, 1993), but in fact is likely to be even more robust thanks to the simple power-law spectra exhibited by GRB afterglows.

In this paper we introduce an objective Swift sample, appropriate for determining the $z_{\text {mean }}$ of GRBs, estimate the fraction of high-redshift bursts and an upper limit on the fraction of heavily obscured afterglows. We also compare the redshift distribution of the sample to models, predicted before the availability of these results, where the GRB rate is proportional to the star formation rate. In addition, we present optical, nearinfrared (NIR) and X-ray observations of the GRB 050814 afterglow and a fit to the resulting spectral energy distribution (SED), allowing us to determine a well constrained redshift of $z=5.3 \pm 0.3$.

\section{Observations}

GRB 050814 was discovered by the Burst Alert Telescope (BAT) aboard the Swift satellite on 14.485 August 2005 UT (Retter et al. 2005). The burst exhibited a slow rise and decline above the background level, with a poorly constrained $t_{90}$ of $65_{-20}^{+40} \mathrm{~s}$ (Tueller et al. 2005). The X-ray Telescope (XRT) slewed promptly to the location and began taking data at $\Delta t=138 \mathrm{~s}$, where $\Delta t$ is the time from the onset of the burst, revealing a fading X-ray source (Morris et al. 2005). Observations with the UV/Optical Telescope began at $\Delta t=167 \mathrm{~s}$ but failed to reveal an optical afterglow (OA) candidate to a limiting magnitude of $V=20.5 \mathrm{mag}$ (Blustin et al. 2005 b). A marginally $(2 \sigma)$ detected OA candidate was reported by Cenko (2005) based on observations at the Palomar 60-inch telescope.

We observed the GRB 050814 XRT error circle in VRI with the Andalucía Faint Object Spectrograph and Camera (ALFOSC) on the Nordic Optical Telescope (NOT) at $\Delta t \approx 13.5 \mathrm{~h}$. A point-like object was detected displaying a red $R-I$ colour, its absence in the $V$-band suggesting a highredshift origin (Jensen et al. 2005). Additional $I$-band imaging

\footnotetext{
${ }^{1}$ However, due to time dilation, little decrease is expected in the spectral energy flux in a given frequency band and at a fixed time of observation after the GRB with increasing redshift (Lamb \& Reichart 2000).
}

Table 1. A $\log$ of the GRB 050814 follow-up imaging observations. Limiting magnitudes are $2 \sigma$ in a circular aperture with a radius equal to the seeing. No correction for Galactic extinction has been applied to the photometry. Note that the NOT and INT $R$-band filters are different, resulting in a slight magnitude discrepancy due to the Ly $\alpha$ break presence in this band.

\begin{tabular}{rcccr}
\hline $\begin{array}{c}\Delta t^{a} \\
\text { [days] }\end{array}$ & $\begin{array}{c}\text { Telescope/ } \\
\text { Instrument }\end{array}$ & Magnitude & $\begin{array}{c}\text { Seeing } \\
{[\operatorname{arcsec}]}\end{array}$ & $\begin{array}{c}\text { Exp. time } \\
{[\mathrm{s}]}\end{array}$ \\
\hline$V$-band: & & & & \\
0.555 & NOT/ALFOSC & $>25.2$ & 0.9 & $3 \times 300$ \\
$R$-band: & & & & \\
0.432 & INT/WFC & $23.09 \pm 0.21$ & 1.2 & 300 \\
0.488 & INT/WFC & $23.09 \pm 0.23$ & 1.4 & 300 \\
0.569 & NOT/ALFOSC & $23.42 \pm 0.09$ & 0.9 & $3 \times 300$ \\
0.584 & INT/WFC & $23.09 \pm 0.23$ & 1.6 & 300 \\
$I$-band: & & & & \\
0.584 & NOT/ALFOSC & $20.55 \pm 0.04$ & 0.9 & 600 \\
1.475 & NOT/ALFOSC & $21.90 \pm 0.08$ & 0.8 & $2 \times 500$ \\
20.375 & NOT/MOSCA & $>24.7$ & 0.8 & $9 \times 400$ \\
$J$-band: & & & & \\
2.769 & UKIRT/UTFI & $22.40 \pm 0.38$ & 0.7 & $64 \times 30$ \\
$K$-band: & & & & \\
0.935 & UKIRT/UTFI & $17.60 \pm 0.03$ & 0.6 & $45 \times 20$ \\
2.737 & UKIRT/UTFI & $20.02 \pm 0.12$ & 0.7 & $80 \times 20$ \\
\hline
\end{tabular}

${ }^{a}$ Days after 14.485 August 2005 UT.

was secured at the NOT using both ALFOSC and the MOSaic CAmera (MOSCA), confirming the fading behaviour of the OA candidate. Supplementary $R$-band imaging was obtained at the Isaac Newton Telescope (INT) using the Wide Field Camera (WFC). All the data were de-biased, flatfield corrected and combined using standard methods. The instrumental optical magnitudes were transformed to the Johnson photometric system using observations of Landolt (1992) standards. The journal of the observations including the results of our photometry is presented in Table 1 .

NIR observations were pursued at the United Kingdom Infrared Telescope (UKIRT) using the UKIRT Fast Track Imager (UFTI) at $\Delta t \approx 22 \mathrm{~h}(K)$ and $\Delta t \approx 66 \mathrm{~h}(J K)$. The data were pipeline processed through the ORACDR system (Cavanagh et al. 2003) to produce flattened, dark- and sky-subtracted mosaic images. Flux calibration was performed against the standard star FS141.

We have also analysed the Swift/XRT observations of GRB 050814 and used these to complement our optical/NIR dataset. Processed event files from the archive were used and background subtracted spectra and light curves from Windowed Timing (WT) and Photon Counting (PC) modes were produced in a standard way.

\section{Spectral energy distribution of the afterglow}

Our multiband observations of the GRB 050814 afterglow allowed the construction of its SED. We extrapolated the magnitudes to a common epoch, namely the nearly simultaneous epoch of our initial VRI NOT observations $(\Delta t=14 \mathrm{~h})$. This was carried out by taking into account the decay indices (as 


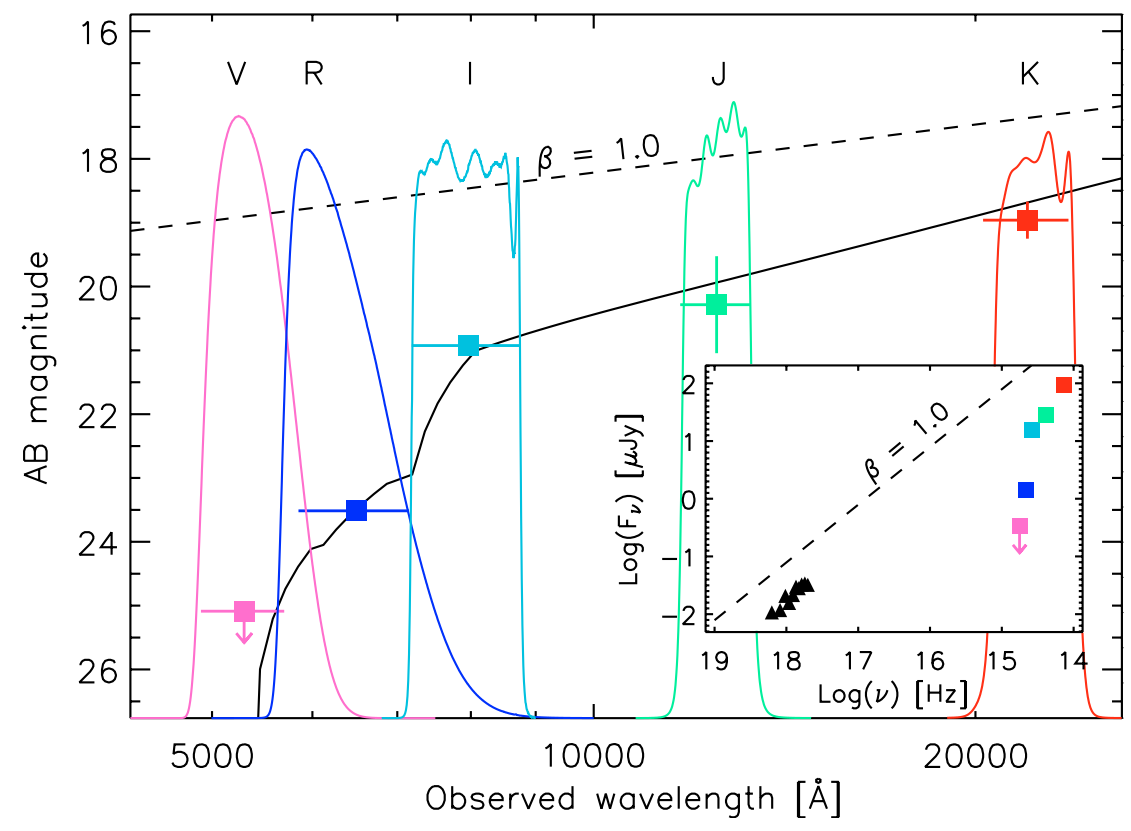

Fig. 1. The spectral energy distribution of the GRB 050814 afterglow at $\Delta t=14 \mathrm{~h}$. The strong break blueward of the $I$-band is too strong to be readily explained by reddening alone and is best fit by the presence of the Ly $\alpha$ break at $z=5.3$. The solid curve is a fit to the data at that redshift. The dashed line shows the spectral slope expected from a synchrotron emission in the fireball model with $\beta=1$. The filter response functions are also shown. The horizontal error bars represent the FWHM of each filter. The $V$-band upper limit is $2 \sigma$. The inset shows the $V R I J K$ observations (filled squares) along with the X-ray spectrum (filled triangles) at $\Delta t=14 \mathrm{~h}$. The dashed line is the same $\beta=1$ slope as in the main panel.

calculated from our photometry in Table 1) $\alpha_{I}=1.34 \pm 0.12$ and $\alpha_{K}=2.08 \pm 0.12\left(F_{v} \propto t^{-\alpha_{v}} v^{-\beta}\right)$. This discrepancy in the decay indices implies a late time $(\Delta t \gtrsim 24 \mathrm{~h})$ rapid decay with $\alpha>2.08$, although the sparse sampling of the light curve means that the timing of this break is poorly constrained. It might be the jet break, a suggestion supported by the fact that the XRT light curve displays a break at approximately $28 \mathrm{~h}$ with a pre-break slope of $\alpha_{\mathrm{X}}=0.58 \pm 0.05$. We have used $\alpha_{\mathrm{X}}$ to obtain lower limits on the extrapolated $J K$ fluxes.

The SED is shown in Fig. 1, where we have corrected the observed data points for foreground (Galactic) extinction using the reddening maps of Schlegel et al. (1998), giving $E(B-V)=0.028 \mathrm{mag}$ at that position on the sky. We note that the flux from the host galaxy has not been subtracted. However, the host is faint enough $(I>24.7 \mathrm{mag})$ that it should contribute less than $2 \%$ of the flux at the epoch we are exploring. The SED has a strong break blueward of the $I$-band, exhibiting colours of $I-K=3.44 \pm 0.29 \mathrm{mag}$ and $R-I=2.87 \pm 0.10 \mathrm{mag}$, corresponding to spectral slopes of $\beta_{I K}=1.78 \pm 0.12$ and $\beta_{R I}=11.70 \pm 0.04$, respectively. The latter value is unreasonable for GRB afterglows, implying an electron energy power-law index more than ten times higher than normally observed. Even in the case of high $A_{V}$, such steep slopes cannot be obtained (see below and also Reichart 2001).

The most likely explanation for the steep break observed is due to the presence of the Ly $\alpha$ break at a redshift of $5<z<6$. To provide a more robust estimate of the GRB 050814 redshift we fit the available photometry at different redshifts, allowing for a range in $\beta$ and $A_{V}$ modelled using the parametrization of Calzetti et al. (2000). The models of Madau (1995) provide the average hydrogen opacity as a function of redshift.

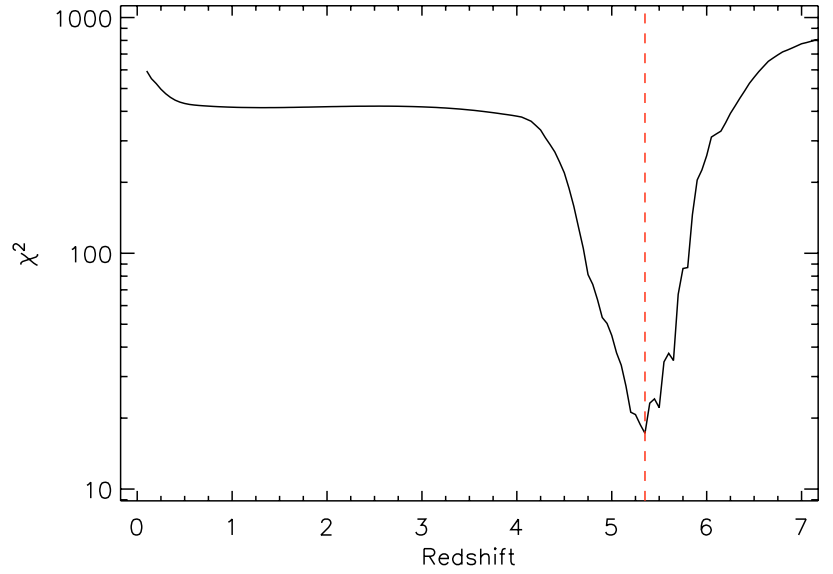

Fig. 2. The calculated $\chi^{2}$ as a function of the GRB 050814 redshift. Low redshifts are ruled out by the data and cannot be fit by extinction. Very high $z>7$ redshifts are ruled out by the presence of the afterglow in the $I$-band. The best fit redshift, indicated by the vertical dashed line, is $z=5.3$ although a range of redshifts from $5.0<z<5.6$ have similar $\chi^{2}$ values.

Figure 2 shows the minimum $\chi^{2}$ for each redshift step plotted against redshift; the best fit is obtained for $z=5.3 \pm 0.3$ and is shown in Fig. 1. However, we are only able to obtain weak constraints on $\beta$ and $A_{V}$. Fixing $\beta=1.0$, a typical value for GRB afterglows, results in a best fit of a restframe $A_{V}=0.9 \mathrm{mag}$ and an unchanged redshift. This $A_{V}$ is marginally higher than has been inferred from the SEDs of preSwift bursts with bright OAs $\left(A_{V}=0.09 \mathrm{mag}\right.$ in GRB 000301C: Jensen et al. 2001; $A_{V}=0.18 \mathrm{mag}$ in GRB 000926: Fynbo et al. 2001; $A_{V}=0.08 \mathrm{mag}$ in GRB 011211: Jakobsson et al. 2003; 
$A_{V}<0.20 \mathrm{mag}$ in GRB 020124: Hjorth et al. 2003b; $A_{V}=$ $0.26 \mathrm{mag}$ in GRB 021004: Holland et al. 2003; $A_{V}<0.50 \mathrm{mag}$ in GRB 030323: Vreeswijk et al. 2004; $A_{V}=0.34 \mathrm{mag}$ in GRB 030429: Jakobsson et al. 2004a) but is a necessary consequence of the red $I-K$ colour. The extrapolated $\beta=1.0$ line, normalized for $A_{V}=0.9 \mathrm{mag}$, slightly overestimates the predicted X-ray flux (inset of Fig. 1), indicating that $\beta$ is a bit steeper; $\beta=1.1$ would make the $\mathrm{X}$-ray data fall on the line. Since the best fit X-ray spectral index is consistent with the assumed optical/NIR one, a cooling break between the optical and X-rays can be ruled out.

We have also explored whether the observed SED could be explained by a lower redshift and a large extinction assuming the three extinction laws given by Pei (1992) for the Milky Way (MW), Large Magellanic Cloud (LMC) and Small Magellanic Cloud (SMC). This was done in the following way. We have calculated the observed $E(R-I) \equiv A_{R}-A_{I}=2.41 \pm 0.10 \mathrm{mag}$ based on $\beta=1.0$ (see above). By (blue)shifting the $R$ and $I$ filters along the aforementioned extinction laws, we can estimate which redshifts produce the observed $E(R-I)$. Using these redshifts we can then compute the predicted $E(I-K)$ and compare to the observed value of $0.88 \pm 0.29 \mathrm{mag}$. In every case the predicted values are $E(I-K)>3.0 \mathrm{mag}$, implying that none of the extinction laws can give rise to the observed SED at a low redshift. In other words, the spectral break between the observed $R$ - and $I$-bands combined with the shallower slope between $I$ and $K$ is inconsistent with the MW, SMC and LMC extinction laws at any lower redshift. Dust close to the GRB site might not follow these extinction laws, for example due to dust being destroyed by the prompt radiation of the GRB itself (e.g. Perna et al. 2003). However, that would tend to produce greyer dust (small grains being preferentially removed), thus reducing rather than increasing the amount of reddening.

The X-ray limits on $N_{\mathrm{H}}$ from the XRT also support a highredshift origin for the red optical/NIR SED, by providing an upper limit on the possible absorbing extragalactic oxygen column density along the line of sight. The values of $N_{\mathrm{H}}$ we derive from the fitting process are sensitive to the fit at low energies. We have used ancillary response files for the WT mode data, generated by xrtmkarf with "inarffile = NONE", which are better at reproducing the low energy continua. The $N_{\mathrm{H}}$ limits using this response file are slightly higher than using the default ancillary response.

We can then obtain a limit on $A_{V}$ using a Galactic empirical relation stating that $A_{V}=1 \mathrm{mag}$ corresponds to $N_{\mathrm{H}}=$ $1.79 \times 10^{21} \mathrm{~cm}^{-2}$ (Predehl \& Schmitt 1995). This implies that for redshifts of $z=(0.5,1.0,2.0,3.0)$ the respective $3 \sigma$ upper limits on $A_{V}$ are $(0.5,0.8,1.8,3.6) \mathrm{mag}$. None of these are large enough to simultaneously reproduce the observed $R-I$ and $I-K$ colours at low redshifts.

\section{The redshift distribution of Swift bursts}

At the time of this writing (30 September 2005) Swift has been operating for approximately 9 months and has detected a total of 70 long-duration GRBs. In order to study the redshift distribution of GRBs, it is important to carefully select a sample containing bursts which have "observing conditions" favorable for redshift determination. Our first criterion is small error circles, hence the bursts have to be localised with the XRT (56 GRBs). In addition we require the Galactic extinction in the direction to the burst to be sufficiently small or $A_{V}^{\mathrm{Gal}}<0.5 \mathrm{mag}$ ( $38 \mathrm{GRBs}$ ). Thirdly, the XRT error circle should be distributed quickly for a relatively rapid follow-up. Although the automatic slewing of Swift was enabled in the middle of January 2005, part of the following month was dedicated to calibration which could not be interrupted. Therefore, we have only included bursts occurring after 1 March 2005 (31 GRBs). Finally, we have rejected (3) bursts with a declination unsuitable (above $+70^{\circ}$ or below $-70^{\circ}$ ) for follow-up observations.

The sample, containing $28 \mathrm{GRBs}$, is presented in Table 2. For each burst we have also listed the Sun-to-field distance $\left(\theta_{\text {Sun }}\right)$, the Moon-to-field distance $\left(\theta_{\text {Moon }}\right)$ and the Moon illumination at the time of the burst. This is done to examine if these parameters affect the redshift determination significantly, e.g. a full Moon close to a burst location. This is of course difficult to quantify as the OA brightness also plays a part. For example, GRB 050820A has a measured redshift even if it occurred during a full Moon and $\theta_{\text {Moon }}=34^{\circ}$. Therefore, we decided not to limit the sample further. This relatively "clean" sample has a very high redshift recovery rate of almost $60 \%$ $(16 / 28)$.

Figure 3 shows the redshift distribution of the 16 bursts with a reported redshift in our Swift sample ${ }^{2}$. Both the mean and the median is $z \approx 2.75$, more than twice as large as the corresponding numbers for pre-Swift bursts (1.37 and 1.04, respectively, calculated from a sample of 42 bursts). A natural explanation for this increase is the lower trigger threshold of Swift compared to previous missions, giving rise to fainter (Swift events are on average $1.7 \mathrm{mag}$ fainter in the $R$-band at a similar epoch: Berger et al. 2005b) and higher redshift bursts. This is complemented by the accurate positions provided by Swift and the rapid response of a variety of telescopes aimed at redshift determinations.

This Swift sample is the most uniform to date and it is of interest to compare its redshift distribution to models predicting the fraction of GRBs expected to occur at a given redshift. Natarajan et al. (2005, hereafter N05) have modelled the expected redshift distribution for GRBs, utilising several models including those which follow the globally averaged star formation rate (model II), and those which scale according to the average metallicity of the Universe at a given redshift (model IV, see e.g. Fynbo et al. 2003; Fryer \& Heger 2005). Gorosabel et al. (2004, hereafter G04) have also carried out a similar exercise, where the GRB rate is assumed to be proportional to the star formation rate. These models are plotted in Fig. 3.

It is remarkable how similar the observed Swift redshift distribution is to the model predictions; we can now reason that GRBs trace star formation (see also Jakobsson et al. 2005a). However, with the available sample and the limited flux sensitivity of the Swift/BAT for $z>5$ bursts, it is currently not possible to determine if GRBs are unbiased tracers of star formation. For example, models II and IV from N05 are nearly

\footnotetext{
2 Note that the sample contains all Swift bursts with a reported redshift except GRB $050126(z=1.29)$.
} 
Table 2. A list of all long-duration GRBs which have a Galactic extinction $A_{V}^{\mathrm{Gal}}<0.5 \mathrm{mag}$, a declination between $-70^{\circ}$ and $+70^{\circ}$, and are localised with the Swift/XRT after 1 March 2005. Here $\theta_{\text {Sun }}$ is the Sun-to-field distance, $\theta_{\text {Moon }}$ the Moon-to-field distance and $I_{\text {Moon }}$ the Moon illumination at the time the burst occurred. For a burst detected in the optical but without a reported redshift, an upper redshift limit is estimated based on the filter it is detected in. References are [1] Kelson \& Berger (2005); [2] Berger \& Mulchaey (2005); [3] Fynbo et al. (2005a); [4] Watson et al. (2005a); [5] Rol et al. (2005); [6] Cenko et al. (2005b); [7] Cenko et al. (2005a); [8] Berger et al. (2005a); [9] Foley et al. (2005); [10] Berger \& Becker (2005); [11] Tanvir et al. (2005); [12] Poole et al. (2005); [13] Starling et al. (2005); [14] Blustin et al. (2005a); [15] Fynbo et al. (2005b); [16] This work; [17] Prochaska et al. (2005); [18] Fynbo et al. (2005c); [19] Kawai et al. (2005); [20] Fugazza et al. (2005); [21] Bloom (2005); [22] Jakobsson et al. (2005b).

\begin{tabular}{|c|c|c|c|c|c|c|c|c|c|c|c|c|c|}
\hline$\overline{\text { GRB }}$ & $\bar{z}$ & $\begin{array}{c}A_{V}^{\mathrm{Gal}} \\
{[\mathrm{mag}]}\end{array}$ & $\begin{array}{r}\theta_{\text {Sun }} \\
{[\mathrm{deg}]}\end{array}$ & $\begin{array}{l}\theta_{\text {Moon }} \\
\text { [deg] }\end{array}$ & $\begin{array}{r}I_{\text {Moon }} \\
{[\%]}\end{array}$ & Ref. & GRB & $\bar{z}$ & $\begin{array}{c}A_{V}^{\mathrm{Gal}} \\
{[\mathrm{mag}]}\end{array}$ & $\begin{array}{r}\theta_{\text {Sun }} \\
\text { [deg] }\end{array}$ & $\begin{array}{l}\theta_{\text {Moon }} \\
{[\mathrm{deg}]}\end{array}$ & $\begin{array}{r}I_{\text {Moon }} \\
{[\%]}\end{array}$ & $\overline{\text { Ref. }}$ \\
\hline 050315 & 1.95 & 0.16 & 59 & 114 & 24 & 1 & 050730 & 3.97 & 0.17 & 84 & 150 & 31 & $\overline{13}$ \\
\hline 050318 & 1.44 & 0.06 & 64 & 83 & 52 & 2 & 050801 & $<2.0$ & 0.32 & 82 & 127 & 15 & 14 \\
\hline 050319 & 3.24 & 0.04 & 132 & 44 & 61 & 3 & 050802 & 1.71 & 0.07 & 79 & 102 & 9 & 15 \\
\hline 050401 & 2.90 & 0.22 & 122 & 36 & 62 & 4 & 050803 & & 0.25 & 136 & 113 & 4 & \\
\hline 050406 & $<3.5$ & 0.07 & 59 & 58 & 10 & 5 & 050814 & 5.3 & 0.09 & 99 & 74 & 60 & 16 \\
\hline 050412 & & 0.07 & 159 & 121 & 11 & & 050819 & & 0.40 & 132 & 59 & 99 & \\
\hline 050416A & 0.65 & 0.10 & 145 & 69 & 44 & 6 & 050820A & 2.61 & 0.15 & 147 & 34 & 100 & 17 \\
\hline $050502 \mathrm{~B}^{a}$ & $<8.5$ & 0.10 & 98 & 177 & 42 & 7 & 050822 & & 0.05 & 105 & 63 & 93 & \\
\hline 050505 & 4.27 & 0.07 & 90 & 130 & 13 & 8 & 050824 & 0.83 & 0.12 & 129 & 17 & 77 & 18 \\
\hline 050525 & 0.61 & 0.32 & 121 & 57 & 98 & 9 & 050904 & 6.29 & 0.21 & 143 & 145 & 0 & 19 \\
\hline 050603 & 2.82 & 0.09 & 56 & 39 & 15 & 10 & 050908 & 3.34 & 0.08 & 146 & 151 & 16 & 20 \\
\hline 050714B & & 0.18 & 67 & 25 & 44 & & 050915A & $<13.0$ & 0.09 & 93 & 109 & 86 & 21 \\
\hline 050716 & $<8.0$ & 0.37 & 108 & 125 & 64 & 11 & 050922B & & 0.12 & 171 & 57 & 80 & \\
\hline 050726 & $<5.0$ & 0.21 & 88 & 147 & 73 & 12 & 050922C & 2.20 & 0.34 & 138 & 93 & 80 & 22 \\
\hline
\end{tabular}

${ }^{a}$ The OA was detected in $I$ but not $V$, suggesting a high redshift (Cenko et al. 2005a).

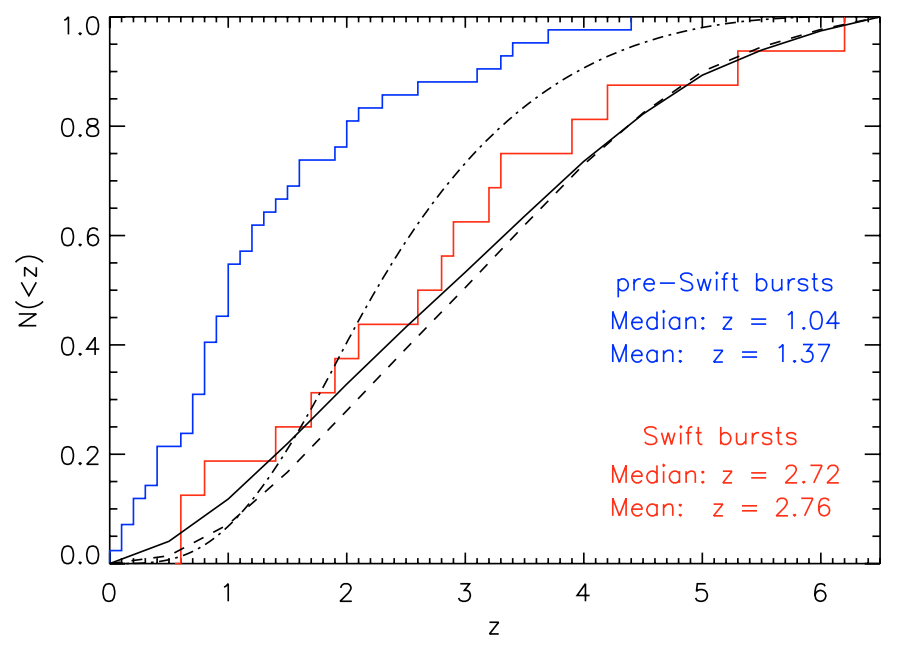

Fig. 3. The cumulative fraction of GRBs as a function of redshift for 42 pre-Swift bursts (upper stepwise curve) and 16 Swift bursts (lower stepwise curve). Overplotted are three simple models for the expectation of the redshift distribution of GRBs: model II from N05 in which the GRB rate is proportional to the star formation rate (solid curve), model IV from N05 in which the GRB rate increases with decreasing metallicity (dashed curve) and a model from G04 in which the GRB rate is proportional to the star formation rate (dash-dotted curve). All three models fold in the Swift/BAT flux sensitivity.

indistinguishable when comparing to the relatively small sample of 16 bursts. Note that although model II from N05 and the G04 model both presuppose that the GRB rate is proportional to the star formation rate, they use different assumptions regarding the poorly determined GRB luminosity function and the intrinsic spectral shape, explaining their difference in Fig. 3.

By including all the bursts in Table 2 we are able to constrain the number of bursts above a specific redshift. For example, $7 \%-40 \%$ of the bursts are located at $z>5$. The N05 and G04 predictions are 10\% and 2\%, respectively, suggesting that the GRB luminosity function parameters and/or the GRB spectral index assumed in N05 might be more appropriate. Bromm \& Loeb (2005) also predict that $10 \%$ of the Swift GRBs should originate at $z>5$.

\section{Discussion and conclusions}

The mean redshift of our relatively unbiased Swift sample $\left(z_{\text {mean }}=2.8\right)$ is larger than the mean redshift of sub-mm galaxies $\left(z_{\text {mean }}=2.4\right.$ : Chapman et al. 2003) and is similar to that of Type 2 AGNs ( $z_{\text {mean }} \sim 3$ : Padovani et al. 2004). With two $z>5$ GRBs discovered within a space of a month, and primarily due to the spectroscopic redshift of $z=6.29$ for GRB 050904 (Kawai et al. 2005), we are finally accessing the GRB high-redshift regime. Are we starting to probe the era of Pop III stars? If the transition between the dark ages and the era of reionization occurred around $z \approx 6-7$ (see e.g. Miralda-Escudé 2003 for a review), the answer might be positive. However, Abel et al. (2002) have calculated that at most one massive metal-free star forms per pre-galactic halo, and since the GRB progenitor needs to be a member of a close binary system in the collapsar scenario (e.g. Fryer et al. 1999; MacFadyen \& Woosley 1999; Zhang et al. 2004), it seems unlikely that Pop III stars could end their lives as GRBs. Woosley \& Heger (2005) have proposed a non-binary possibility in the 
collapsar scenario, introducing unusually rapidly rotating massive stars. It is therefore conceivable that Pop III stars are GRB progenitors, although it is evident that the number of unknowns is currently too large to arrive at a concrete conclusion.

The number of GRBs without an OA in our sample, sets a strict upper limit on the fraction of heavily dust obscured afterglows as expected in ultraluminous infrared host galaxies (ULIRGs). At most 20\% (6/28) of the GRB hosts in the sample can be of this nature. In fact, 5 of the non-detections only have a relatively shallow optical limit, typically $V \gtrsim 19$ mag (albeit early post-burst), suggesting that the fraction is possibly lower. This is consistent with results from sub-mm observations of pre-Swift burst (e.g. Tanvir et al. 2004).

The sample contains GRB 050814, whose OA was particularly faint in the $R$-band; the observed optical to X-ray spectral slope is flatter $\left(\beta_{\mathrm{OX}}=0.36\right)$ than expected for the fireball model. Hence, GRB 050814 is classified as a dark burst as defined by Jakobsson et al. (2004b). We have argued that this is most likely due to the high-redshift nature $(z=5.3)$ of this burst; the $R-I$ colour is extremely red which is impossible to explain by strong extinction given the observed $I-K$ colour. Indeed, a similar conclusion was proposed for GRB 980329 (Fruchter 1999).

It is clear that GRBs have now opened up a window to the very high-redshift Universe. The emerging GRB redshift histogram (Fig. 3) strongly indicates that they can be used to trace the star formation in the Universe over a wide redshift range $(0 \lesssim z \lesssim 7)$. Future instrumentation, such as the X-shooter (D'Odorico et al. 2004), will hopefully shed light on the end of the dark ages and the possible GRB/Pop III connection.

Acknowledgements. We thank Dale A. Frail and the anonymous referee for excellent comments. P.J., B.L.J. and K.P. acknowledge support from the Instrument Centre for Danish Astrophysics (IDA). R.P. acknowledges PPARC for support, while A.L. and N.R.T. thank PPARC for support through postdoctoral and senior research fellowships, respectively. The research of J.G. is supported by the Spanish Ministry of Science and Education through programmes ESP200204124-C03-01 and AYA2004-01515. J.M.C.C. gratefully acknowledges partial support from IDA and the NBI's International Ph.D. School of Excellence. The Dark Cosmology Center is funded by the Danish National Research Foundation. The authors acknowledge benefits from collaboration within the EU FP5 Research Training Network "Gamma-Ray Bursts: An Enigma and a Tool". Based on observations made with the Nordic Optical Telescope (Isaac Newton Telescope), operated on the island of La Palma jointly by Denmark, Finland, Iceland, Norway, and Sweden (the Isaac Newton Group), in the Spanish Observatorio del Roque de los Muchachos of the Instituto de Astrofísica de Canarias.

\section{References}

Abel, T., Bryan, G. L., \& Norman, M. L. 2002, Science, 295, 93 Berger, E., \& Becker, G. 2005, GCN Circ., 3520

Berger, E., \& Mulchaey, J. 2005, GCN Circ., 3122

Berger, E., Kulkarni, S. R., Bloom, J. S., et al. 2002, ApJ, 581, 981

Berger, E., Cenko, S. B., Steidel, C., Reddy, N., \& Fox, D. B. 2005a, GCN Circ., 3368

Berger, E., Kulkarni, S. R., Fox, D. B., et al. 2005b, ApJ, 634, 501

Bloom, J. S. 2005, GCN Circ., 3990
Blustin, A., Band, D., Hunsberger, S., et al. 2005a, GCN Circ., 3733

Blustin, A., Retter, A., Marshall, F., Chester, M., \& Gehrels, N. 2005b, GCN Circ., 3804

Bromm, V., \& Loeb, A. 2005, ApJ, submitted [arXiv:astro-ph/0509303]

Calzetti, D., Armus, L., Bohlin, R. C., et al. 2000, ApJ, 533, 682

Cavanagh, B., Hirst, P., Jenness, T., et al. 2003, ASPC, 295, 237

Cenko, S. B. 2005, GCN Circ., 3807

Cenko, S. B., Fox, D. B., Rich, J., et al. 2005a, GCN Circ., 3357

Cenko, S. B., Kulkarni, S. R., Gal-Yam, A., \& Berger, E. 2005b, GCN Circ., 3542

Chapman, S., Blain, A. W., Ivison, R. J., \& Smail, I. R. 2003, Nature, 422,695

Christensen, L., Hjorth, J., \& Gorosabel, J. 2004, A\&A, 425, 913

D’Odorico, S., Andersen, M. I., Conconi, P., et al. 2004, SPIE, 5492, 220

Foley, R. J., Chen, H.-W., Bloom, J., \& Prochaska, J. X. 2005, GCN Circ., 3483

Fruchter, A. S. 1999, ApJ, 512, L1

Fruchter, A. S., Pian, E., Thorsett, S. E., et al. 1999, ApJ, 516, 683

Fryer, C. L., \& Heger, A. 2005, ApJ, 623, 302

Fryer, C. L., Woosley, S. E., \& Hartmann, D. H. 1999, ApJ, 526, 152

Fugazza, D., Fiore, F., Patat, N., et al. 2005, GCN Circ., 3948

Fynbo, J. P. U., Gorosabel, J., Dall, T. H., et al. 2001, A\&A, 373, 796

Fynbo, J. P. U., Jakobsson, P., Møller, P., et al. 2003, A\&A, 406, L63

Fynbo, J. P. U., Hjorth, J., Jensen, B. L., et al. 2005a, GCN Circ., 3136

Fynbo, J. P. U., Sollerman, J., Jensen, B. L., et al. 2005b, GCN Circ., 3749

Fynbo, J. P. U., Jensen, B. L., Sollerman, J., et al. 2005c, GCN Circ., 3874

Gehrels, N., Chincarini, G., Giommi, P., et al. 2004, ApJ, 611, 1005

Ghirlanda, G., Ghisellini, G., Lazzati, D., \& Firmani, C. 2004, ApJ, 613, L13

Gorosabel, J., Lund, N., Brandt, S., Westergaard, N. J., \& Castro Cerón, J. M. 2004, A\&A, 427, 87 (G04)

Haislip, J., Nysewander, M. C., Reichart, D., et al. 2005, Nature, submitted [arXiv: astro-ph/0509660]

Hjorth, J., Sollerman, J., Møller, P., et al. 2003a, Nature, 423, 847

Hjorth, J., Møller, P., Gorosabel, J., et al. 2003b, ApJ, 597, 699

Holland, S. T., Weidinger, M., Fynbo, J. P. U., et al. 2003, AJ, 125, 2291

Jakobsson, P., Hjorth, J., Fynbo, J. P. U., et al. 2003, A\&A, 408, 941

Jakobsson, P., Hjorth, J., Fynbo, J. P. U., et al. 2004a, A\&A, 427, 785

Jakobsson, P., Hjorth, J., Fynbo, J. P. U., et al. 2004b, ApJ, 617, L21

Jakobsson, P., Björnsson, G., Fynbo, J. P. U., et al. 2005a, MNRAS, 362,245

Jakobsson, P., Fynbo, J. P. U., Paraficz, D., et al. 2005b, GCN Circ., 4029

Jensen, B. L., Fynbo, J. U., Gorosabel, J., et al. 2001, A\&A, 370, 909

Jensen, B. L., Fynbo, J. P. U., Hjorth, J., et al. 2005, GCN Circ., 3809

Kawai, N., Kosugi, G., Aoki, K., et al. 2005, Nature, in press [arXiv:astro-ph/0512052]

Kelson, D., \& Berger, E. 2005, GCN Circ., 3101

Lamb, D. Q., \& Reichart, D. E. 2000, ApJ, 536, 1

Landolt, A. U. 1992, AJ, 104, 340

MacFadyen, A. I., \& Woosley, S. E. 1999, ApJ, 524, 262

Madau, P. 1995, ApJ, 441, 18

Miralda-Escudé, J. 2003, Science, 300, 1904

Morris, D. C., Burrows, D. N., Kennea, J. A., Racusin, J. L., \& Gehrels, N. 2005, GCN Circ., 3805

Mörtsell, E., \& Sollerman, J. 2005, JCAP, 6, 9

Natarajan, P., Albanna, B., Hjorth, J., et al. 2005, MNRAS, 364, L8 (N05) 
Padovani, P., Allen, M. G., Rosati, P., \& Walton, N. A. 2004, A\&A, 424,545

Pei, Y. C. 1992, ApJ, 395, 130

Perna, R., Lazzati, D., \& Fiore, F. 2003, ApJ, 585, 775

Poole, T., Moretti, A., Holland, S. T., et al. 2005, GCN Circ., 3698

Predehl, P., \& Schmitt, J. H. M. M. 1995, A\&A, 293, 889

Price, P. A., Cowie, L. L., Minezaki, T., et al. 2005, ApJL, submitted [arXiv: astro-ph/0509697]

Prochaska, J. X., Bloom, J. S., Wright, J. T., et al. 2005, GCN Circ., 3833

Reichart, D. E. 2001, ApJ, 553, 235

Retter, A., Barbier, L., Barthelmy, S., et al. 2005, GCN Circ., 3799

Rol, E., Schady, P., Hunsberger, S., et al. 2005, GCN Circ., 3186

Schlegel, D. J., Finkbeiner, D. P., \& Davis, M. 1998, ApJ, 500, 525

Spinrad, H., Stern, D., Bunker, A., et al. 1998, AJ, 116, 2617

Stanek, K. Z., Matheson, T., Garnavich, P. M., et al. 2003, ApJ, 591, L17

Starling, R. L. C., Vreeswijk, P. M., Ellison, S. L., et al. 2005, A\&A, 442, L21
Steidel, C. C., \& Hamilton, D. 1992, AJ, 104, 941

Steidel, C. C., \& Hamilton, D. 1993, AJ, 105, 2017

Tagliaferri, G., Antonelli, L. A., Chincarini, G., et al. 2005, A\&A, 443, L1

Tanvir, N. R., Barnard, V. E., Blain, A. W., et al. 2004, MNRAS, 352, 1073

Tanvir, N., Lowe, K., Gledhill, T., et al. 2005, GCN Circ., 3632

Tueller, J., Markwardt, C., Barbier, L., et al. 2005, GCN Circ., 3803

Vreeswijk, P. M., Ellison, S. L., Ledoux, C., et al. 2004, A\&A, 419, 927

Watson, D., Fynbo, J. P. U., Ledoux, C., et al. 2005a, ApJ, submitted [arXiv: astro-ph/0510368]

Watson, D., Reeves, J. N., Hjorth, J., et al. 2005b, ApJL, in press [arXiv: astro-ph/0509640]

Weymann, R. J., Stern, D., Bunker, A., et al. 1998, ApJ, 505, L95

Woosley S. E., \& Heger, A. 2005, ApJ, submitted [arXiv: astro-ph/0508175]

Zhang, W., Woosley, S. E., \& Heger, A. 2004, ApJ, 608, 365 